\title{
Short communication \\ Quantification of angiogenic growth factors released by human dental cells after injury
}

\author{
L. Tran-Hung, P. Laurent, J. Camps, I. About* \\ Laboratoire IMEB-ERT 30, Faculté d’Odontologie, Université de la Méditerranée, 27 Boulevard Jean Moulin, \\ 13355 Marseille Cedex 05, France
}

\begin{abstract}
A B S T R A C T
Objective: Angiogenesis is a key step in the dental pulp healing sequence which involves the dentine bridge formation. In a previous work, we showed that dental pulp cells secrete soluble factors which interact with endothelial cells and affect the process of angiogenesis. The objective of this work was to quantify the angiogenic growth factors released by mechanically injured human dental pulp cells and the effect of 2-hydroxyethyl methacrylate (HEMA) on this secretion.

Design: Pulp cells were prepared from immature third molars explants by the outgrowth method. Cell monolayers were either subjected to mechanical injuries or treated with increasing concentrations of HEMA. ELISA was used to quantify the secreted angiogenic growth factors in the culture media after different time periods of injury and after incubation with different concentrations of HEMA.

Results: Pulp cells secreted significant levels of PDGF-AB, VEGF and FGF-2. The concentration of these factors increased shortly $(5 \mathrm{~h}$ ) after injury and returned to initial values after 1 day. HEMA treatment increased VEGF secretion but decreased that of FGF-2 in a dose-dependent manner while it did not affect PDGF-AB level.

Conclusions: Dental pulp cells secrete angiogenic growth factors which play a pivotal role in angiogenesis which precedes the reparative dentine formation. PDGF-AB seems to play a major role because its level showed the highest increase in mechanically injured cells. The presence of HEMA affects both FGF-2 and VEGF levels and may partially explain the lack of dentinebridgingafter directpulpcapping with an adhesivesystem.
\end{abstract}

\section{Introduction}

Angiogenesis is a critical step of wound healing. This process is regulated by interplay of numerous growth factors including platelet-derived growth factor (PDGF), vascular endothelial growth factor (VEGF) and basic fibroblast growth factor (FGF-2). Among these, VEGF is considered as an essential factor for the differentiation of the vascular system ${ }^{1}$; FGF-2 is known to stimulate angiogenesis in vivo and plays a significant role in the neovascularisation of damaged or traumatised tissue ${ }^{2}$; while PDGF is thought to play important roles in the process of embryogenesis and tissue regeneration. ${ }^{3}$

The dental pulp is a highly vascularised tissue and has a naturally high inherent regenerative potential. Several lines of evidence suggest the presence of progenitor or postnatal stem cells in the pulp capable of differentiation into 
odontoblast-like cells and secretion of reparative dentine in vitro. $^{4,5}$ It is well established that injured endothelial cells release signalling molecules (FGFs 1, 2 and 4) which are involved in the initiation of the inflammatory reaction and the healing process $^{6}$ and seem to be involved in the recruitment of odontoblast-like cells at the injury site. ${ }^{7}$ Among the factors released after injury, FGF-2 is involved in angiogenesis and may act as a mitogen for pulp progenitor cells. ${ }^{8}$ It stimulates the proliferation of rat immature dental pulp cells and has an inductive activity on mineralised calcium production in $3 \mathrm{D}$ cultures of dental pulp cells in collagen type I gel. ${ }^{9}$

In vitro studies have demonstrated that most adhesive system components had cytotoxic effects on cultured mammalian fibroblasts. ${ }^{10-12}$ 2-Hydroxy-ethyl methacrylate (HEMA) is the major component released from resin-modified glass ionomer cements and dental bonding agents. ${ }^{13}$ This monomer affects the differentiation of human pulp cells into odontoblast-like cells as demonstrated by the inhibition of odontoblasts specific proteins expression and the inhibition of mineral nodule formation by dental pulp cells in long term cultures. ${ }^{14}$ However, when applied under conditions similar to the clinical situation as a component of bonding agents, this effect disappears after complete photopolymerisation. ${ }^{15}$ It is known that direct pulp capping of human teeth with an adhesive system induces a local increase in blood vessel density ${ }^{16}$ and a lack of dentine bridge formation. ${ }^{17-20}$ However, the molecular mechanisms involved in this phenomenon are not well elucidated.

The dentine matrix act as a reservoir of growth factors and it has been reported that a high concentration of PDGF and lower concentrations of VEGF and FGF-2 are sequestered in the human dentine. The release of these factors from the dentine matrix under pathological conditions such as severe carious lesions could make an important contribution to the overall reparative response of the dentine-pulp complex. ${ }^{21}$ In a previous work, we showed that pulp cells act on angiogenesis by secreting soluble factors. Neutralizing both FGF-2 and VEGF was not enough to inhibit the angiogenic effect completely, suggesting that other factor(s) may be involved in this process. ${ }^{22}$

In the present work, we hypothesised that in addition to its effects on the specific functions and mineralisation potential of pulp cells, HEMA may also affect pulp cells secretion of angiogenic growth factors which play a key role in pulp healing and dentine bridge formation.

The objective of this work was to quantify the angiogenic growth factors released by mechanically injured human dental pulp cells and the effect of HEMA on this event.

\section{Materials and methods}

Human pulp cells were prepared from immature third molars as described previously. ${ }^{5}$ Briefly, the teeth were obtained from 16 year-old adolescents in compliance with the French legislation (informed patients and parents consent and Institutional Review Board approval of the protocol used). Ten teeth from five individuals (two teeth/subject) were used and each tooth was treated as a distinct sample for the subsequent studies. After the extraction process, the teeth were washed and their apical part was removed. The dental pulp was minced and the resulting small explants were cultured in 100-mm-diameter culture dishes (Becton Dickinson Labware, Lincoln Park, NJ). Confluent cultures were collected by trypsinisation and sub cultured. Cells were cultured in 60-mm-diameter culture dishes (Becton Dickinson Labware, Lincoln Park, NJ) in Minimum essential medium supplemented with $10 \%$ fetal bovine serum, $2 \mathrm{mM}$ glutamine, $100 \mathrm{UI} / \mathrm{ml}$ penicillin, $100 \mu \mathrm{m}$ streptomycin and $0.25 \mu \mathrm{g} / \mathrm{ml}$ amphotericin B (Fungizone).

Injuries to pulp cells were performed mechanically on 60$\mathrm{mm}$-diameter culture dishes. Three series were performed on cells from passage 3, 4 and 5 of each tooth. Ten straight lines of $3 \mathrm{~cm}$ length per dish were performed with sterile scalpels to disrupt the cell monolayer. The media obtained after a contact period of 5 h, 1 day, 2 day and 3 days with injured or intact cells were used for the quantification of angiogenic factors.

\subsection{HEMA treatment}

Pulp cells cultured in 60-mm-diameter culture dishes were incubated with HEMA in MEM medium for 1 day at the following concentrations: 10,50 and $100 \mu \mathrm{M}$. Three series were performed as described above. The conditioned media were then used for the quantification of angiogenic factors.

\subsection{Sandwich enzyme linked immunosorbent assay (ELISA)}

The quantification of platelet-derived growth factor (PDGF$A B)$, basic fibroblast growth factor (FGF-2) and vascular endothelial growth factor (VEGF), and was performed using the sandwich enzyme linked immunosorbent assay (Quantikine Cytokine ELISA kit, R\&D system, France). Briefly, the media samples were incubated in microwell plates precoated with monoclonal antibodies to either PDGF-BB, VEGF or FGF-2. Serial dilutions of recombinant standards were also incubated to generate the standard curves for each growth factor. After $2 \mathrm{~h}$, the wells were washed with the buffer solution and were then incubated with peroxidase-conjugated polyclonal antibodies to either PDGF-AA, VEGF or FGF-2 for 2 h. After washing, the wells were incubated for $30 \mathrm{~min}$ with the enzyme substrate solution before adding the stop solution. The optical density was determined using a microplate reader (Metertech Inc., Taipei, Taiwan) at a wavelength of $450 \mathrm{~nm}$.

\subsection{Statistical analysis}

Each experiment was done in triplicate on each tooth and each series was performed on a different passage number. Error bars reflect the standard deviation and probability values were assessed using the Mann-Whitney non-parametric text. $p<0.05$ was considered significant.

\section{Results}

\subsection{Pulp cells secretion of PDGF-AB, VEGF and FGF-2 increased after injury}

Intact human pulp cells released high levels of angiogenic growth factors: PDGF-AB $(169.69 \pm 20.15 \mathrm{pg} / \mathrm{ml})$, VEGF 


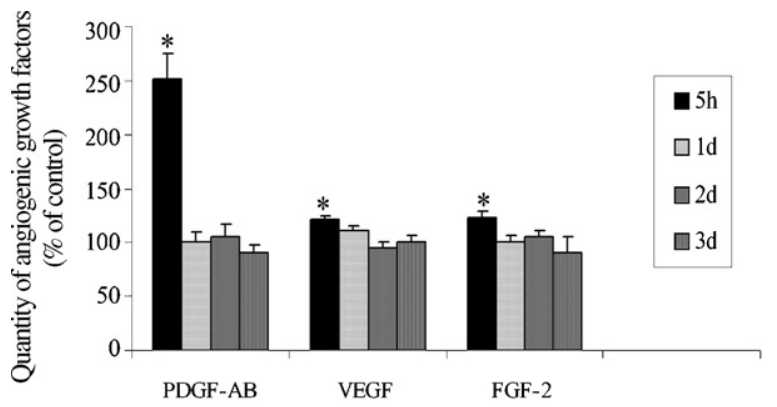

Fig. 1 - Dental pulp cells secretion of angiogenic growth factors after injury. Injuries were performed with sterile scalpels (10 straight lines, $3 \mathrm{~cm}$ long each) on confluent pulp cells cultures and the media were collected after $5 \mathrm{~h}, 1$ day, 2 day or 3 days of injury to quantify the released angiogenic factors. PDGF-AB, FGF-2 and VEGF secretion was quantified by ELISA $(n=3)$. The secretion of these factors increased after $5 \mathrm{~h}$ of injury and returned to initial values after 1 day. PDGF-AB secretion $5 \mathrm{~h}$ after injury increased up to 2.5 times that of intact cells. The results were expressed as percentage of controls (without injury). * indicates a significant difference with the control $(p<0.05)$.

(769.97 $\pm 20.89 \mathrm{pg} / \mathrm{ml})$ and FGF-2 (299.37 $\pm 31.89 \mathrm{pg} / \mathrm{ml})$. Injured pulp cells showed a significant increase of released growth factors as compared to intact cells. This increase was of $150.59 \%$ for PDGF-AB, 20.6\% for VEGF and 23.35\% for FGF-2. This increase was obtained $5 \mathrm{~h}$ after injury and then returned to initial values. There was no significant difference in the concentration of these factors as compared to controls after 1, 2 or 3 days of injury (Fig. 1).

\subsection{Effect of HEMA on PDGF-AB, VEGF and FGF-2 secretion}

When the pulp cells were subjected to increasing concentrations of HEMA, differential effects on angiogenic growth factors were observed. While there was almost no effect on PDGF-AB levels, the secretion of VEGF increased. This effect was dose-dependent: while there was no significant difference between the control and HEMA at $10 \mu \mathrm{M}$, the monomer increased VEGF secretion to $30.69 \%$ at $50 \mu \mathrm{M}(p<0.05)$ and up to $49.42 \%(p<0.05)$ at $100 \mu \mathrm{M}$.

By contrast, HEMA treatment decreased FGF-2 secretion in a dose-dependent manner. While there was no significant difference in FGF-2 secretion with HEMA at $10 \mu \mathrm{M}$. FGF-2 secretion dropped to $26.22 \%(p<0.05)$ at $50 \mu \mathrm{M}$ and reached $39.62 \%(p<0.05)$ with HEMA at $100 \mu \mathrm{M}$ (Fig. 2).

\section{Discussion}

The initial steps of dentinogenesis including angiogenesis play an important role in the pulp healing sequence after injury. It has been shown that human perivascular progenitor/stem cells can be activated and proliferated in 1 day in response to

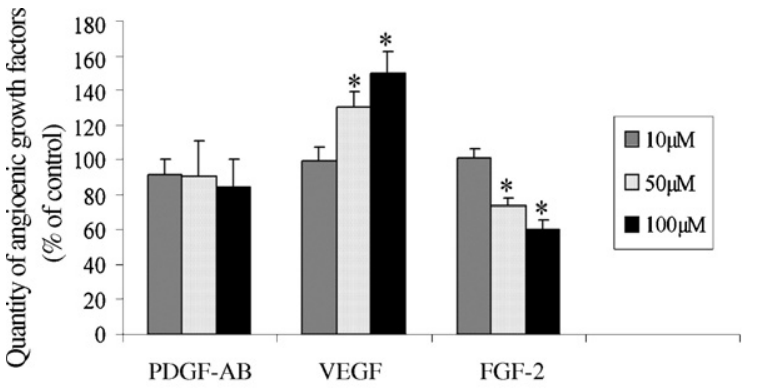

Fig. 2 - Effect of HEMA on pulp cells angiogenic growth factors secretion. Pulp cells were incubated with HEMA at 10,50 or $100 \mu \mathrm{M}$. The media were collected after 1 day of incubation to quantify the released angiogenic factors by ELISA $(n=3)$. Increasing concentrations of HEMA did not affect PDGF-AB level. They increased VEGF secretion but decreased that of FGF-2 both in a dose-dependent manner. The results were expressed as percentage of control (without HEMA treatment). * indicates a significant difference with the control $(p<0.05)$.

pulp injury. After this proliferation, these cells migrate to the injury site in 2 weeks. ${ }^{23}$ The increase of neovascularisation is a very important event for the pulp healing and it is in agreement with the release of angiogenic growth factors from the pulp following orthodontic forces. ${ }^{24}$ Our results suggest that angiogenesis precedes these steps which are necessary for the formation of reparative dentine.

The present study clearly demonstrated that human pulp cells secrete PDGF-AB, VEGF and FGF-2, particularly after injury. The release of these factors was very rapid ( $5 \mathrm{~h}$ after injury) and corresponds well to the pathological changes in the pulp following injury. The level of these factors returned to initial values after 1 day. This is a confirmation of our previous work which showed that pulp cells express VEGF and FGF-2 and that these molecules exert their angiogenic effects as soluble factors. ${ }^{22}$ The return to initial values only after 1 day confirms their very early potential effects on angiogenesis.

It has been reported that angiogenic growth factors are sequestered within the dentine matrix with high concentrations of PDGF-AB (570 pg/mg of dentine), lower concentrations of VEGF $(63 \mathrm{pg} / \mathrm{mg})$ and FGF-2 $(40 \mathrm{pg} / \mathrm{mg}) \cdot{ }^{21}$ This high concentration of PDGF-AB is suggestive of an important role of this growth factor in the dentine-pulp biology. In our work, the highest concentration was that of VEGF. However, the highest increase after injury, as compared to the control, was that of PDGF-AB. This high secretion of PDGF-AB can explain the persistence of angiogenesis after neutralisation of FGF-2 and VEGF in our previous study ${ }^{22}$ and highlights its role in angiogenesis during pulp healing after injury. Additionally, PDGF-AB has been found to inhibit the formation of dentinelike nodule in vitro. ${ }^{25}$ In the context of reparative dentine synthesis, the very high increase of PDGF-AB shortly (5 h) after injury may allow angiogenesis to proceed before any mineralisation. These findings place emphasis on the important role of PDGF-AB in the repair process of damaged dental pulp. No stimulation in PDGF-AB secretion was observed in the presence of HEMA in our work. The absence of this stimulation 
may affect the angiogenesis which is required for reparative dentine formation.

In this study, we showed an increase of VEGF secretion in human pulp cells after HEMA treatment. VEGF up-regulation and secretion has been recently reported in mouse odontoblast-like cells in response to HEMA after 1 day. ${ }^{26}$ Similar results were obtained in situations simulating pathological conditions such as lipoteichoic acid application. ${ }^{27}$ The enhanced VEGF secretion may explain the increase in pulp vascularisation observed when adhesive resins are used in direct pulp capping. ${ }^{16}$ This phenomenon may be beneficial in pulp healing processes to protect the pulp tissue against pathological conditions but may equally be critical to maintain vascular homeostasis since the pulp chamber is inextensible. In vivo, anti-angiogenic factors such as angiostatin and endostatin are also expressed by dental pulp cells that may counteract the VEGF up-regulation caused by the adhesive resin. ${ }^{26}$

By contrast, HEMA decreased pulp cells FGF-2 secretion. This growth factor is essential for odontoblast differentiation of immature pulp cells and induces the expression of mineralised tissue-related proteins in particular conditions such as 3D formation of dental cells in collagen type I gel. ${ }^{9}$ Additionally, FGF-2 is a mitogen factor that is involved in dental pulp fibroblast proliferation ${ }^{9}$ and the recruitment of skin progenitor fibroblasts at the injury site. ${ }^{8}$ The reduced FGF2 secretion observed in our work may affect the proliferation and the migration of progenitor pulp cells. This event may explain the lack of dentine bridging observed in the majority of human teeth treated after direct pulp capping with adhesive resins. The lack of dentine bridging might render the pulp more susceptible to inflammation mediated by bacterial contamination if microleakage is observed particularly at the resin-tooth interface. ${ }^{16}$

Under pathological conditions, angiogenesis is a key step in the healing sequence of the dental pulp with hard tissue formation. Injured pulp cells secrete angiogenic growth factors to stimulate angiogenesis which precedes the reparative dentine formation. The presence of HEMA disturbs this process and may partially explain the lack of dentine bridging in direct pulp capping with an adhesive system.

\section{Acknowledgements}

This work was supported by institutional funding from the French "Ministère de l'éducation nationale, de l'enseignement supérieur et de la recherche" and from the program "Bonus Qualite Recherche-Université" of the Université de la Méditerranée to Imad ABOUT. The authors wish to thank Dr. JeanCharles Gardon for providing the third molars used in this work.

\section{RE F E R E N C E S}

1. Ferrara N. Vascular endothelial growth factor. Eur J Cancer 1996;32:2413-22.

2. Gerwins P, Skoldenberg E, Claesson-Welsh L. Function of fibroblast growth factors and vascular endothelial growth factors and their receptors in angiogenesis. Crit Rev Oncol Hematol 2000;34:185-94.

3. Matsuoka J, Grotendorst GR. Two peptides related to platelet-derived growth factor are present in human wound fluid. Proc Natl Acad Sci USA 1989;86:4416-20.

4. Gronthos S, Mankani M, Brahim J, Robey PG, Shi S. Postnatal human dental pulp stem cells (DPSCs) in vitro and in vivo. Proc Natl Acad Sci USA 2000;97:13625-30.

5. About I, Bottero MJ, de Denato P, Camps J, Franquin JC, Mitsiadis TA. Human dentin production in vitro. Exp Cell Res 2000;258:33-41.

6. Martin P. Wound healing-aiming for perfect skin regeneration. Science 1997;276:75-81.

7. Mathieu S, El-Battari A, Dejou J, About I. Role of injured endothelial cells in the recruitment of human pulp cells. Arch Oral Biol 2005;50:109-13.

8. Nugent MA, Iozzo RV. Fibroblast growth factor-2. Int J Biochem Cell Biol 2000;32:115-20.

9. Nakao K, Itoh M, Tomita Y, Tomooka Y, Tsuji T. FGF-2 potently induces both proliferation and DSP expression in collagen type I gel cultures of adult incisor immature pulp cells. Biochem Biophys Res Commun 2004;325:1052-9.

10. Hanks CT, Strawn SE, Wataha JC, Craig RG. Cytotoxic effects of resin components on cultured mammalian fibroblasts. J Dent Res 1991;70:1450-5.

11. Ratanasathien S, Wataha JC, Hanks CT, Dennison JB. Cytotoxic interactive effects of dentin bonding components on mouse fibroblasts. J Dent Res 1995;74:602-6.

12. Geurtsen W, Lehmann F, Spahl W, Leyhausen G. Cytotoxicity of 35 dental resin composite monomers/ additives in permanent $3 \mathrm{~T} 3$ and three human primary fibroblast cultures. J Biomed Mater Res 1998;41:474-80.

13. Nakabayashi N, Takarada K. Effect of HEMA on bonding to dentin. Dent Mater 1992;8:125-30.

14. About I, Camps J, Mitsiadis TA, Bottero MJ, Butler W, Franquin JC. Influence of resinous monomers on the differentiation in vitro of human pulp cells into odontoblasts. J Biomed Mater Res 2002;63:418-23.

15. About I, Camps J, Burger AS, Mitsiadis TA, Butler WT, Franquin JC. Polymerized bonding agents and the differentiation in vitro of human pulp cells into odontoblastlike cells. Dent Mater 2005;21:156-63.

16. Costa CA, Mesas AN, Hebling J. Pulp response to direct capping with an adhesive system. Am J Dent 2000;13:81-7.

17. do Nascimento AB, Fontana UF, Teixeira HM, Costa CA. Biocompatibility of a resin-modified glass-ionomer cement applied as pulp capping in human teeth. Am J Dent 2000;13:28-34.

18. Hebling J, Giro EM, Costa CA. Biocompatibility of an adhesive system applied to exposed human dental pulp. $J$ Endod 1999;25:676-82.

19. Pereira JC, Segala AD, Costa CA. Human pulpal response to direct pulp capping with an adhesive system. Am J Dent 2000;13:139-47.

20. Silva GA, Lanza LD, Lopes-Junior N, Moreira A, Alves JB. Direct pulp capping with a dentin bonding system in human teeth: a clinical and histological evaluation. Oper Dent 2006;31:297-307.

21. Roberts-Clark DJ, Smith AJ. Angiogenic growth factors in human dentine matrix. Arch Oral Biol 2000;45:1013-6.

22. Tran-Hung L, Mathieu S, About I. Role of human pulp fibroblasts in angiogenesis. J Dent Res 2006;85:819-23.

23. Tecles O, Laurent P, Zygouritsas S, Burger AS, Camps J, Dejou J, et al. Activation of human dental pulp progenitor/ stem cells in response to odontoblast injury. Arch Oral Biol 2005;50:103-8.

24. Derringer KA, Linden RW. Vascular endothelial growth factor, fibroblast growth factor 2, platelet-derived growth factor and transforming growth factor beta released in 
human dental pulp following orthodontic force. Arch Oral Biol 2004;49:631-41.

25. Yokose S, Kadokura H, Tajima N, Hasegawa A, Sakagami H, Fujieda K, et al. Platelet-derived growth factor exerts disparate effects on odontoblast differentiation depending on the dimers in rat dental pulp cells. Cell Tissue Res 2004;315:375-84.
26. Mantellini MG, Botero T, Yaman P, Dennison JB, Hanks CT, Nor JE. Adhesive resin and the hydrophilic monomer HEMA induce VEGF expression on dental pulp cells and macrophages. Dent Mater 2006;22:434-40.

27. Telles PD, Hanks CT, Machado MA, Nor JE. Lipoteichoic acid up-regulates VEGF expression in macrophages and pulp cells. J Dent Res 2003;82:466-70. 